ASSER International Sports Law Series 
More information about this series at http://www.springer.com/series/8858 
Leanne O'Leary

\section{Employment and Labour Relations Law in the Premier League, NBA and International Rugby Union}




\section{Leanne O'Leary}

Faculty of Law and Criminology

Edge Hill University

Ormskirk

Lancashire, UK

ISSN 1874-6926

ISSN 2215-003X (electronic)

ASSER International Sports Law Series

ISBN 978-94-6265-158-6

ISBN 978-94-6265-159-3 (eBook)

DOI 10.1007/978-94-6265-159-3

Library of Congress Control Number: 2016954705

Published by T.M.c. ASSER PRESS, The Hague, The Netherlands www.asserpress.nl

Produced and distributed for T.M.C. ASSER PRESS press by Springer-Verlag Berlin Heidelberg

(C) T.M.C. ASSER PRESS and the author 2017

No part of this work may be reproduced, stored in a retrieval system, or transmitted in any form or by any means, electronic, mechanical, photocopying, microfilming, recording or otherwise, without written permission from the Publisher, with the exception of any material supplied specifically for the purpose of being entered and executed on a computer system, for exclusive use by the purchaser of the work.

The use of general descriptive names, registered names, trademarks, service marks, etc. in this publication does not imply, even in the absence of a specific statement, that such names are exempt from the relevant protective laws and regulations and therefore free for general use.

Printed on acid-free paper

This Springer imprint is published by Springer Nature

The registered company is Springer Science+Business Media B.V.

The registered company address is: Van Godewijckstraat 30,3311 GX Dordrecht, The Netherlands 
...that the law's chief concern about the use of power is not who is exercising it but what the power is and whom it affects.

Stephen Sedley (2000) Public power and private power. In: Forsythe $\mathrm{C}$ (ed) Judicial review and the constitution.

Hart Publishing, Oxford, p 306 


\section{Series Information}

Books in the ASSER International Sports Law Series comprehensibly chart and analyse legal and policy developments in the emerging field of European and international sports law. Within scholarly publishing, the series is the most cited in its area and uniquely features contributions from the leading sports law scholars. It is a valuable resource for practitioners, academics, sports officials, and anyone interested in or impacted by sports and law.

This Series is developed, edited and published by the ASSER International Sports Law Centre in The Hague. The Centre's mission is to provide a centre of excellence, in particular by providing high-quality research, services and products to the sporting world at large (sports ministries, international-intergovernmental - organisations and federations, the professional sports industry, etc.) on both a national and an international basis. As a pioneer in the field of European and international sports law, the Centre has established a worldwide network of academics and practitioners and cooperation agreements with various sports law institutes and centres in Europe and beyond.

Apart from the Series, the Centre edits and publishes The International Sports Law Journal.

\section{Editor-in-chief}

Prof. Dr. Ben Van Rompuy

e-mail: b.van.rompuy@law.leidenuniv.nl

\section{Series editor}

Dr. Antoine Duval

e-mail: a.duval@asser.nl

\section{Editorial Office}

ASSER International Sports Law Centre

T.M.C. Asser Instituut

P.O. Box 30461

2500 GL The Hague

The Netherlands

www.sportslaw.nl

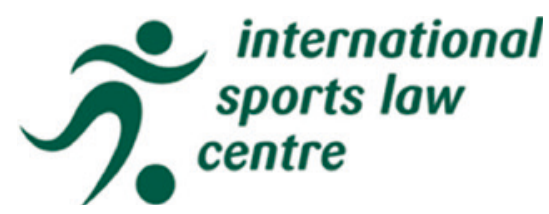




\section{Acknowledgements}

I would like to express my sincere gratitude to the following organizations for their time and valued assistance during the completion of this book: The Football Association, The Premier League, The Professional Footballers' Association, World Rugby and the International Rugby Players' Association. I would also like to thank the editors Prof. Dr. Ben Van Rompuy and Dr. Antoine Duval for their invaluable comments and my colleagues, friends and family in New Zealand and elsewhere. 


\section{Contents}

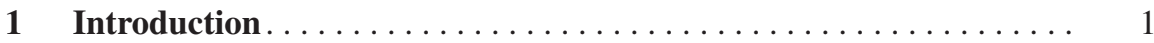

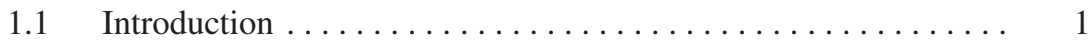

1.2 The Legal Status of an Athlete.................. 3

1.3 The Special Nature of an Employment Contract.......... 4

1.4 Employment and Labour Relations Law ............... 7

1.5 Economic Characteristics of a Team Sports Competition ....... 9

1.6 Regulation of Sport ...................... 12

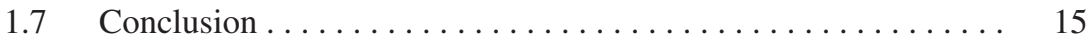

References............................. 17

2 History of Labour Relations and Working Conditions in the Premier League, NBA and Rugby Union . . . . . . . . . . . . . 19

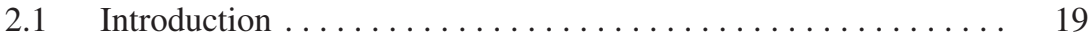

$2.2 \quad$ Premier League Football $\ldots \ldots \ldots \ldots \ldots \ldots \ldots \ldots \ldots \ldots \ldots$

$2.3 \quad$ National Basketball Association $\ldots \ldots \ldots \ldots \ldots \ldots \ldots \ldots . \ldots \ldots$

2.4 International Rugby Union. . . . . . . . . . . . . . . . . . . . . 42

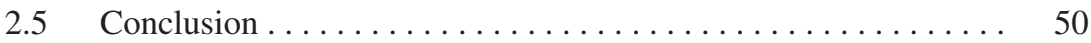

References.............................. 51

3 Organization and Regulation of the Premier League,

NBA and Rugby Union . . . . . . . . . . . . . . . . . . . 53

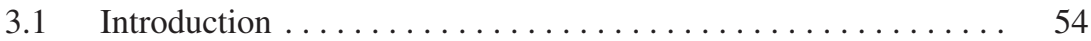

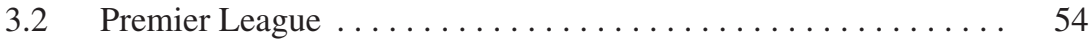

3.2.1 Premier League Clubs . . . . . . . . . . . . . . . . . . 57

3.2.2 Competition Format .................. 58

$3.2 .3 \quad$ Football League ........................ 58

3.2.4 Players and the Professional Footballers' Association. . . 59

3.2.5 Regulatory Framework. .................. 61

$3.3 \quad$ National Basketball Association $\ldots \ldots \ldots \ldots \ldots \ldots \ldots \ldots 68$

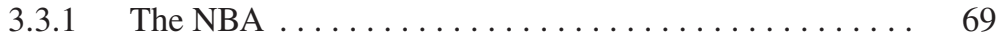

3.3.2 NBA Commissioner...................... 71

$3.3 .3 \quad$ NBA Teams $\ldots \ldots \ldots \ldots \ldots \ldots \ldots \ldots \ldots \ldots \ldots, 72$ 
3.3.4 Competition Format.................... 73

3.3.5 Players and the National Basketball Players' Association......................... 74

3.3.6 Fédération Internationale de Basketball and USA Basketball.......................... 75

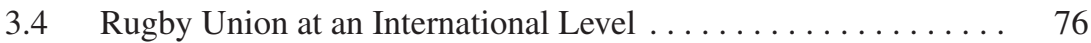

3.4.1 World Rugby ....................... 76

3.4.2 National Federations ..................... 81

3.4.3 Rugby Union Players and the International Rugby Players' Association .................. 83

3.5 Conclusion . . . . . . . . . . . . . . . . . . . . . . . . . . . 84

References.............................. 85

4 Employment and Labour Relations Law in the United Kingdom and Professional Sport $\ldots \ldots \ldots \ldots \ldots \ldots \ldots \ldots \ldots \ldots \ldots \ldots$

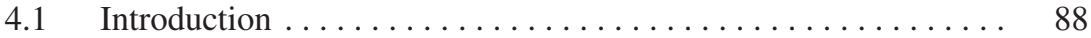

4.2 The Employment Contract...................... 89

4.2.1 Concept of Worker ..................... 91

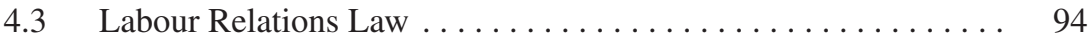

4.3.1 Trade Union and Labour Relations (Consolidation)

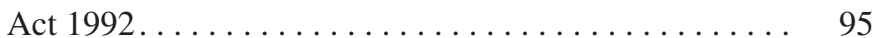

4.4 Common Law Doctrine of Restraint of Trade . . . . . . . . . . . 104

4.5 Conclusion ............................... 111

References............................... 112

5 Free Movement of Athletes in the Sports Industry ............ 115

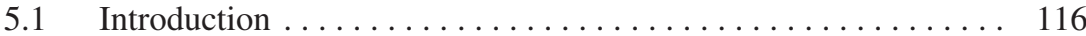

$5.2 \quad$ Free Movement of Workers . . . . . . . . . . . . . . 118

5.2.1 Direct Discrimination, Indirect Discrimination and Non-discriminatory Measures/Obstacles to Free Movement . . . . . . . . . . . . . . . . . . . . . 120

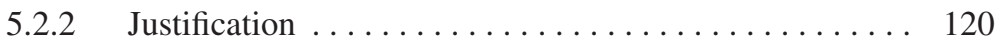

5.2.3 Freedom of Movement in the Sports Industry ....... . 121

$5.3 \quad$ Free Movement of the Self-employed ................. 129

5.3.1 Direct Discrimination, Indirect Discrimination and Non-discriminatory Measures/Obstacles and Justifications . . . . . . . . . . . . . . . . . . . 130

5.3.2 Freedom to Provide Services in the Sports Industry ... . 131

5.4 Conclusion ............................... 134 References................................ 135

6 Labour Relations Law at a European Level and Professional Sport...................................... 137

$6.1 \quad$ Introduction . . . . . . . . . . . . . . . . . . . . 138

6.2 Internal Market Law and Trade Union Activities. . . . . . . . . . . 139 
6.2.1 European Competition Law v Collective Agreements . . . 141

6.2.2 Strike Action v Fundamental Freedoms. . . . . . . . . . . . . 149

6.3 Social Dialogue . . . . . . . . . . . . . . . . . . . . 154

6.3.1 Sectoral Social Dialogue Committee for Professional Football . . . . . . . . . . . . . . 157

6.3.2 Sectoral Social Dialogue Committee in Sport and Active Leisure . . . . . . . . . . . . . . . . . 161

6.4 European Convention on Human Rights . . . . . . . . . . . . 163

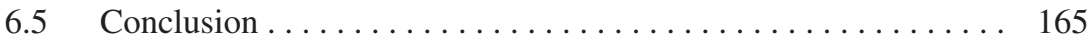

References............................. 166

7 Federal Labour Law in the USA and Professional Sport . . . . . . . . 169

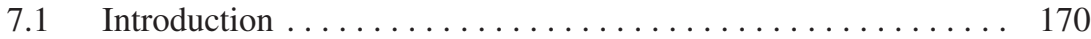

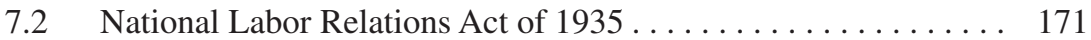

7.2.1 Recognition, Collective Bargaining

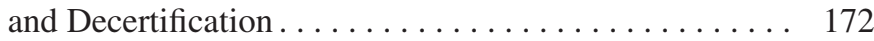

7.2.2 Unfair Labour Practices, Right to Strike and Industrial Action . . . . . . . . . . . . . . . . . 175

7.3 Statutory and Non-statutory Labour Exemptions . . . . . . . . . 178

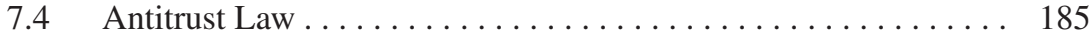

7.4.1 Contract, Combination or Conspiracy . . . . . . . . . 186

7.4.2 Unreasonably Restrains Trade . . . . . . . . . . . . 189

7.4.3 Exemption for the Business of Baseball . . . . . . . . . . 192

7.4.4 Antitrust Cases and Professional Players. . . . . . . . . . 193

7.5 Commissioner's Power. . . . . . . . . . . . . . . . . . . 195

7.5.1 Federal Labour Law and Collective Bargaining . . . . . . 195

7.5.2 Exceeding Authority, Bad Faith and Procedural Unfairness . . . . . . . . . . . . . . . . . . . . . . 197

7.6 Conclusion ......................... 200

References............................ 201

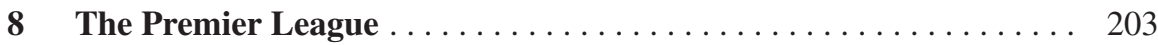

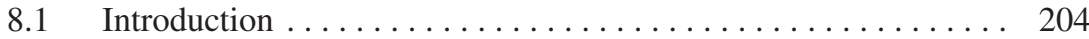

8.2 Role of Collective Bargaining . . . . . . . . . . . . . . . . . . 204

8.2.1 The Professional Football Negotiating

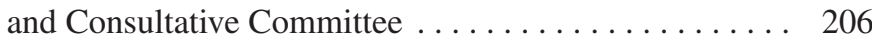

8.2.2 Bargaining Issues . . . . . . . . . . . . . . . . 207

8.2 .3 Collective Agreements . . . . . . . . . . . . . . . 208

8.2 .4 Football Association Role . . . . . . . . . . . . . . 208

8.2 .5 Trade Disputes ....................... 209

8.3 Standard Players' Contract. . . . . . . . . . . . . . . . 210

8.4 Wage Regulation . . . . . . . . . . . . . . . . 214

8.4.1 Short Term Cost Control Rules . . . . . . . . . . . . . . . 216

8.5 Movement of Players . . . . . . . . . . . . . . . . . . . . . . 220

8.5.1 FA Rules: Registration and Transfer ............ 221 
8.5.2 Premier League Rules: Registration and Transfer . . . . . 222

8.5 .3 Transfer Fees . . . . . . . . . . . . . . . . . . 223

8.5.4 International Transfer System . . . . . . . . . . . . . . 224

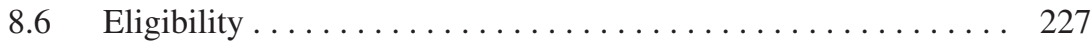

8.6.1 National Immigration Law. . . . . . . . . . . . . . . . . . . 229

8.6.2 Squad List System: Home Grown Players . . . . . . . . . . 230

$8.7 \quad$ Intellectual Property. . . . . . . . . . . . . . . . . . . 234

8.7.1 Standard Players' Contract. . . . . . . . . . . . . 235

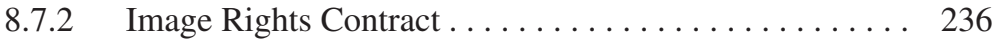

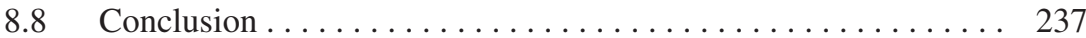

References.............................. 239

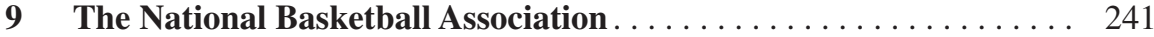

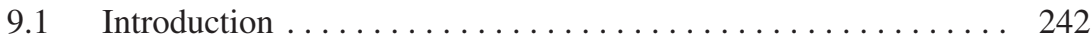

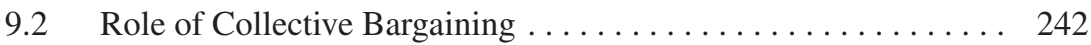

9.2.1 Collective Agreement. . . . . . . . . . . . . . . . . . . 243

9.2.2 Bargaining Issues and Trade Disputes . . . . . . . . . . . 244

9.3 Uniform Players' Contract . . . . . . . . . . . . . . . . . . . 245

9.4 Wage Regulation ....................... 246

9.5 Movement of Players . . . . . . . . . . . . . . . . . . 250

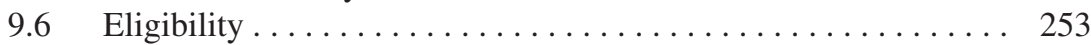

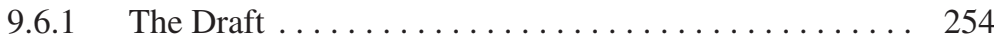

9.6.2 International Transfer Rules. . . . . . . . . . . . . . . . 258

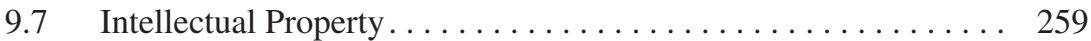

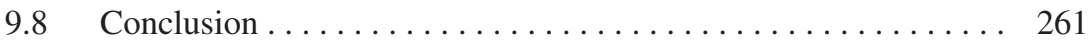

References............................... 262

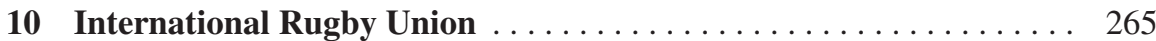

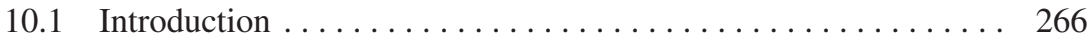

10.2 Role of Collective Bargaining . . . . . . . . . . . . . . 267

10.2.1 Consultation, Issues of Concern and Trade Disputes. . . 270

10.2.2 The Rugby Athlete's Commission . . . . . . . . . . . . 273

10.3 RWC Participation Agreement. . . . . . . . . . . . . . . . . 274

10.4 Wage Regulation ... . . . . . . . . . . . . . . . . . . . 276

10.5 Movement of Players . . . . . . . . . . . . . . . . 276

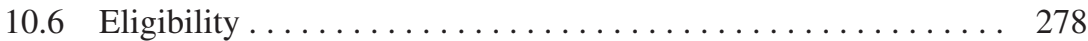

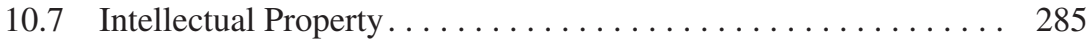

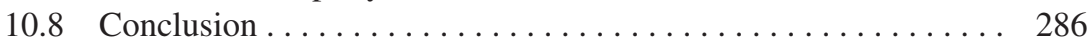

References.......................... 287

11 Decision-Making in a Professional Team Sports Competition . . . . . . 289

11.1 Introduction . . . . . . . . . . . . . . . . . . . . . . 290

11.2 Organization of the Premier League, the NBA

and Rugby Union . . . . . . . . . . . . . . . . . . . . . 292

11.3 Employment Arrangements . . . . . . . . . . . . . . . . . . 294

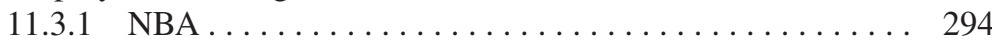


11.3.2 Premier League . . . . . . . . . . . . . . . . . 296

11.3.3 International Rugby Union. . . . . . . . . . . . . . . 300

11.4 Decision-Making in Professional Sport . . . . . . . . . . . . . . . 302

11.4.1 National Leagues . . . . . . . . . . . . . . . . . 302

11.4.2 Competitions Organized at an International Level. . . . . 309

11.5 Conclusion ......................... 313

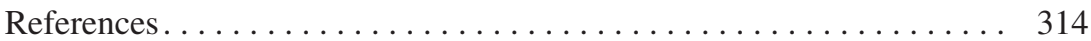

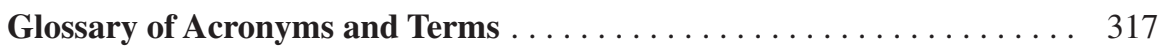

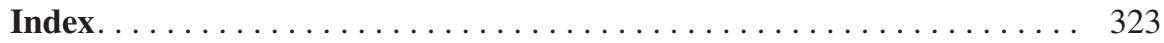

